\title{
Measuring Cubeness of 3D Shapes
}

\author{
Carlos Martinez-Ortiz and Joviša Žunić* \\ Department of Computer Science, University of Exeter, Exeter EX4 4QF, U.K. \\ $\{\mathrm{cm} 265, \mathrm{~J}$.Zunic\}@ex.ac.uk
}

\begin{abstract}
In this paper we introduce a new measure for $3 D$ shapes: cubeness. The new measure ranges over $(0,1]$ and reaches 1 only when the given shapes is a cube. The new measure is invariant with respect to rotation, translation and scaling, and is also robust with respect to noise.
\end{abstract}

Keywords: $3 D$ shape, compactness measure, image processing.

\section{Introduction}

Shape descriptors are a powerful tool for shape classification tasks in $2 D$ and $3 D$. Many shape descriptors are already studied in the literature and used in practice. Some of the best known ones in $2 D$ are: elongation $([10])$, convexity $([5])$, rectangularity $([6)$, rectilinearity $([13)$, sigmoidality $([7)$, circularity $([12])$, etc. There are also some $3 D$ shape descriptors like: compactness $([1])$, geometric moments ([4]), Fourier Transforms ([1] $)$, etc.

In this paper we define a new $3 D$ shape descriptor which measures the similarity of an object and a cube. We call this new measure "cubeness". Notice that the $3 D$ measure $\mathcal{C}_{d}(S)$, presented in [2, is similar in some respect to the measure introduced here: it is maximised by a cube - i.e. $\mathcal{C}_{d}(S)$ picks up the highest possible value (which is 1 ) if and only if the measured shape is cube. Such measure is defined as follows:

$$
\mathcal{C}_{d}(S)=\frac{n(S)-A(S) / 6}{n-(\sqrt[3]{n(S)})^{2}}
$$

where $A(S)$ is the area of the enclosing surface, i.e. the sum of the area of the voxels faces which form the surface of the shape, and $n(S)$ is the number of voxels in the shape.

Measure $\mathcal{C}_{d}(S)$ is a measure of discrete compactness of rigid solids composed of a finite number of polyhedrons. When these polyhedrons are voxels, the most compact shape according to $\mathcal{C}_{d}(S)$ is a cube and thus it was select as a comparable measure to the measure introduced in this paper.

One possible application of the new cubeness measure introduced in this paper can be as an additional feature for $3 D$ search engines like the one presented in [3]. Their search engine uses spherical harmonics to compute similarity measures

* J. Žunić is also with the Mathematical Institute, Serbian Academy of Arts and Sciences, Belgrade.

E. Bayro-Corrochano and J.-O. Eklundh (Eds.): CIARP 2009, LNCS 5856, pp. 716-723, 2009.

(C) Springer-Verlag Berlin Heidelberg 2009 
used for the search. The cubeness measure presented in this paper could be used as an additional similarity measure for such a search engine. Some experiments on this point will be performed in the future.

This paper is organised as follows. The next section introduces the new cubeness measure and highlights several of its desirable properties. Section 3 gives several examples which demonstrate the behaviour of the new measure. Section 4 contains some comments and conclusions.

\section{Cubeness Measure}

In this section we define the new cubeness measure. Throughout this paper we will assume that all appearing shapes have non-empty interior, i.e. they have a strictly positive volume. We will also assume that two shapes $S_{1}$ and $S_{2}$ to be equal if the symmetric set difference $\left(S_{1} \backslash S_{2}\right) \cup\left(S_{2} \backslash S_{1}\right)$ has volume zero. Such assumptions are necessary to keep the proofs mathematically rigorous, but they are not of practical importance - e.g. under these assumptions the open ball $\left\{(x, y, z) \mid x^{2}+y^{2}+z^{2}<1\right\}$ and closed one $\left\{(x, y, z) \mid x^{2}+y^{2}+z^{2} \leq 1\right\}$ are the same shape which is totally acceptable from the view point of image processing and computer vision applications, even that they differ for a spherical surface $\left\{(x, y, z) \mid x^{2}+y^{2}+z^{2}=1\right\}$ (having the volume equal to zero). Also any appearing shape will be considered that its centroid coincides with the origin even if not explicitly stated. $S(\alpha, \beta)$ will denote the shape $S$ rotated along the $X$ axis by an angle $\alpha$ and, along the $Y$ axis by an angle $\beta$. We will use the $l_{\infty}$-distance in our derivation; just a short remainder that $l_{\infty}$-distance between points $A=\left(a_{1}, a_{2}, a_{3}\right)$ and $B=\left(b_{1}, b_{2}, b_{3}\right)$ is defined as:

$$
l_{\infty}(A, B)=\max \left\{\left|a_{1}-b_{1}\right|,\left|a_{2}-b_{2}\right|,\left|a_{3}-b_{3}\right|\right\} .
$$

Trivially, the set of all points $X=(x, y, z)$ whose $l_{\infty}$-distance from the origin $O=(0,0,0)$ is not bigger than $r$ is a cube. Such a cube will be denoted by $\mathcal{Q}(r)$ :

$$
\mathcal{Q}(r)=\left\{X=(x, y, z) \mid l_{\infty}(X, O) \leq r\right\}=\{(x, y, z) \mid \max \{|x|,|y|,|z|\} \leq r\} .
$$

To define the new cubeness measure, we start with the quantity:

$$
\min _{\alpha, \beta \in[0,2 \pi]} \iiint_{S(\alpha, \beta)} \max \{|x|,|y|,|z|\} d x d y d z
$$

and show that such a quantity reaches its minimum value if and only if the shape $S$ is a cube. By exploiting this nice property we will come to a new cubeness measure. First we prove the following theorem:

Theorem 1. Let $S$ be a given shape whose centroid coincides with the origin, and let $S(\alpha, \beta)$ denote the shape $S$ rotated along the $X$ axis by an angle $\alpha$ and along the $Y$ axis by an angle $\beta$. Then, 


$$
\begin{aligned}
& \iiint_{S} \max \{|x|,|y|,|z|\} d x d y d z \\
& \frac{\operatorname{Volume}(S)^{4 / 3}}{\iiint_{S} \max \{|x|,|y|,|z|\} d x d y d z} \\
& \frac{\operatorname{Volume}(S)^{4 / 3}}{8} \Longleftrightarrow \frac{3}{8} \Longleftrightarrow S=\mathcal{Q}\left(\frac{\operatorname{Volume}(S)^{1 / 3}}{2}\right) \\
& \frac{\min _{\alpha, \beta \in[0,2 \pi]} \iiint_{S(\alpha, \beta)} \max \{|x|,|y|,|z|\} d x d y d z}{\operatorname{Volume}(S)^{4 / 3}}=\frac{3}{8} \Longleftrightarrow S \text { is a cube. }
\end{aligned}
$$

Proof. Let $S$ be a shape as in the statement of the theorem. Also, let $\mathcal{Q}$, for short, denote the cube $\mathcal{Q}\left(\frac{\operatorname{Volume}(S)^{1 / 3}}{2}\right)$, i.e. the cube is aligned with the coordinate axes and the faces intersect the axes at points: $(a / 2,0,0),(-a / 2,0,0),(0, a / 2,0)$, $(0,-a / 2,0),(0,0, a / 2)$ and $(0,0,-a / 2)$ and $a=\operatorname{Volume}(S)^{1 / 3}$ (see Fig $1(\mathrm{a})$. Trivially, the volumes of $S$ and $\mathcal{Q}$ are the same, and also:

(i) The volume of the set differences $S \backslash \mathcal{Q}$ and $\mathcal{Q} \backslash S$ are the same, because the volumes of $S$ and $\mathcal{Q}$ are the same;

(ii) The points from $\mathcal{Q} \backslash S$ are closer (with respect to $l_{\infty}$-distance) to the origin than the points from $S \backslash \mathcal{Q}$. More formally: if $(u, v, w) \in S \backslash \mathcal{Q}$ and $(p, q, r) \in$ $\mathcal{Q} \backslash S$, then $\max \{|u|,|v|,|w|\}>\max \{|p|,|q|,|r|\}$ (see. Fig. 1 (b)] and (c).

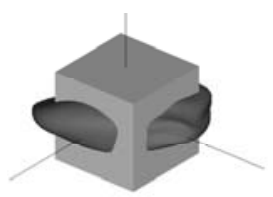

(a) $\mathcal{Q}$ and $S$

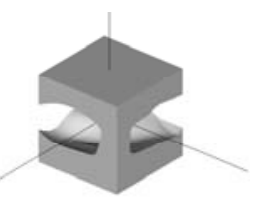

(b) $\mathcal{Q} \backslash S$

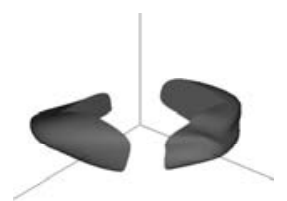

(c) $S \backslash \mathcal{Q}$

Fig. 1. Shapes $S$ and $\mathcal{Q}=\mathcal{Q}\left(\operatorname{Volume}(S)^{1 / 3} / 2\right)$. Both shapes have the same volume. Points in $\mathcal{Q} \backslash S$ are closer to the origin (using $l_{\infty}$-distance) than those in $S \backslash \mathcal{Q}$.

Further (i) and (ii) give:

$$
\iint_{S \backslash \mathcal{Q}} \max \{|x|,|y|,|z|\} d x d y d z \geq \iiint_{\mathcal{Q} \backslash S} \max \{|x|,|y|,|z|\} d x d y d z .
$$

Now, we derive:

$$
\begin{aligned}
& \iiint_{S} \max \{|x|,|y|,|z|\} d x d y d z=8 \iiint_{\substack{(x, y, z) \in S \\
x, y, z \geq 0}} \max \{x, y, z\} d x d y d z \\
= & 48 \iiint_{\substack{(x, y, z) \in S \\
x \geq y \geq z \geq 0}} x d x d y d z=48 \int_{0}^{a / 2} \int_{0}^{x} \int_{0}^{y} x d x d y d z=\frac{3}{8} \cdot a^{4},
\end{aligned}
$$

which proves (5) since $a=\operatorname{Volume}(S)^{1 / 3}$. 
The proof of (6) comes from the fact that equality (8) holds only when the shapes $S$ and $Q$ are the same, i.e. when $\operatorname{Volume}(S \backslash \mathcal{Q})=\operatorname{Volume}(\mathcal{Q} \backslash S)=0$.

To prove (7) let $\alpha_{0}$ and $\beta_{0}$ be the angles which minimise $\iiint_{S} \max \{|x|,|y|,|z|\} d x d y d z$ :

$$
\iiint_{S\left(\alpha_{0}, \beta_{0}\right)} \max \{|x|,|y|,|z|\} d x d y d z=\min _{\alpha, \beta \in[0,2 \pi]} \iiint_{S(\alpha, \beta)} \max \{|x|,|y|,|z|\} .
$$

Since $\operatorname{Volume}(S)=\operatorname{Volume}(S(\alpha, \beta))=\operatorname{Volume}\left(S\left(\alpha_{0}, \beta_{0}\right)\right)$, then (see (6) $)$

$$
\frac{\iiint_{S\left(\alpha_{0}, \beta_{0}\right)} \max \{|x|,|y|,|z|\} d x d y d z}{\operatorname{Volume}\left(S\left(\alpha_{0}, \beta_{0}\right)\right)^{4 / 3}}=\frac{3}{8}
$$

would imply that $S\left(\alpha_{0}, \beta_{0}\right)$ must be equal to $\mathcal{Q}$ - i.e., $S$ must be a cube.

Theorem 1 tells us that $\iiint \max \{|x|,|y|,|z|\} d x d y d z$ reaches its minimum value $S(\alpha, \beta)$

of $3 / 8$ only when $S$ is a cube. Based on this, we give the following definition for the cubeness measure.

Definition 1. The cubeness measure $\mathcal{C}(S)$ of a given shape $S$ is defined as

$$
\mathcal{C}(S)=\frac{3}{8} \cdot \frac{\operatorname{Volume}(S)^{4 / 3}}{\min _{\alpha, \beta \in[0,2 \pi]} \iiint_{S(\alpha, \beta)} \max \{|x|,|y|,|z|\} d x d y d z} .
$$

The following theorem summarizes the desirable properties of $\mathcal{C}(S)$.

Theorem 2. The cubeness measure $\mathcal{C}(S)$ has the following properties:

(a) $\mathcal{C}(S) \in(0,1], \quad$ for all $3 D$ shapes $S$ with non-empty interior;

(b) $\mathcal{C}(S)=1 \Longleftrightarrow S$ is a cube;

(c) $\mathcal{C}(S)$ is invariant with respect to similarity transformations;

Proof. Items (a) and (b) follow directly from Theorem 1

Item (c) follows from the fact that both $\min _{\alpha, \beta \in[0,2 \pi} \iiint_{S(\alpha, \beta)} \max \{|x|,|y|,|z|\} d x d y d z$ and volume of $S$ are rotation invariant, which makes $\mathcal{C}(S)$ rotation invariant. $\mathcal{C}(S)$ is translation invariant by definition, since it is assumed that the centroid of $S$ coincides with the origin. Finally if $S$ is scaled by a factor of $\mathbf{r}$ then easily

$$
\begin{array}{r}
\min _{\alpha, \beta \in[0,2 \pi]} \iiint_{\mathbf{r} \cdot S(\alpha, \beta)} \max \{|x|,|y|,|z|\} d x d y d z \\
=\mathbf{r}^{4} \cdot \min _{\alpha, \beta \in[0,2 \pi]} \iiint_{S(\alpha, \beta)} \max \{|x|,|y|,|z|\} d x d y d z
\end{array}
$$


and

$$
\operatorname{Volume}(\mathbf{r} \cdot S)=\mathbf{r}^{3} \cdot \operatorname{Volume}(S)
$$

and, consequently,

$$
\begin{aligned}
\mathcal{C}(\mathbf{r} \cdot S) & =\frac{3}{8} \cdot \frac{\operatorname{Volume}(\mathbf{r} \cdot S)^{4 / 3}}{\min _{\alpha \in[0,2 \pi], \beta \in[0,2 \pi] \cdot \mathbf{r} S(\alpha, \beta)} \max \{|x|,|y|,|z|\} d x d y d z} \\
& =\frac{3}{8} \cdot \frac{\left(\mathbf{r}^{3} \cdot \operatorname{Volume}(S)\right)^{4 / 3}}{\mathbf{r}^{4} \cdot \min _{\alpha \in[0,2 \pi], \beta \in[0,2 \pi]} \iiint_{S(\alpha, \beta)} \max \{|x|,|y|,|z|\} d x d y d z}=\mathcal{C}(S)
\end{aligned}
$$

which means that $\mathcal{C}(S)$ is scale invariant.

The cubeness measure $\mathcal{C}(S)$ is very similar in spirit to the compactness measure presented in [14:

$$
\mathcal{K}(S)=\frac{3^{5 / 3}}{5(4 \pi)^{2 / 3}} \cdot \frac{\mu_{0,0,0}(S)^{5 / 3}}{\mu_{2,0,0}(S)+\mu_{0,2,0}(S)+\mu_{0,0,2}(S)} .
$$

however they differ in the distance measure used: $\mathcal{K}(S)$ uses euclidean distance while $\mathcal{C}(S)$ uses $l_{\infty}$-distance. Therefore $\mathcal{C}(S)$ is indeed a form of compactness maximized by a cube. $\mathcal{C}_{d}(S)$ is also a compactness measure maximized by a cube, while a sphere is the most compact shape according to $\mathcal{K}(S)$.

\section{$3 \quad$ Experiments Illustrating $\mathcal{C}(S)$ Measure}

In this section we give several examples in order to illustrate the behaviour of $\mathcal{C}(S)$. Figure 2 shows several geometric shapes ranked according to $\mathcal{C}(S)$ measure. $\mathcal{C}(S)$ is given under each figure. The values in brackets corresponds to $C_{d}(S)$. Notice that $C_{d}(S)$ measure also reaches it maximum value 1 for the cube. It is in accordance with our expectation that shapes which are more "spread out", like (e), have lower $\mathcal{C}(S)$ measure. Another fact which is worth pointing out is that the values obtained by $\mathcal{C}(S)$, for the shapes displayed, are in a wider range $[0.340-1.000]$ than values computed by $C_{d}(S)$ which are in the range

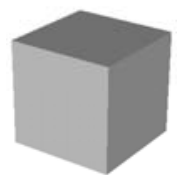

(a) 1.000

(1.000)

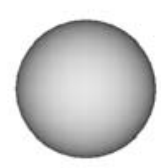

(b) 0.970

(0.995)

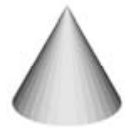

(c) 0.812

(0.734)

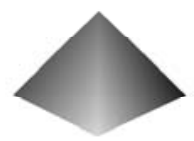

(d) 0.795

(0.754)

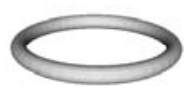

(e) 0.340

(0.785)

Fig. 2. Different geometric shapes ordered according to their cubeness $\mathcal{C}(S)$. The values $\mathcal{C}_{d}(S)$ are in brackets. 
$[0.734-1.000]$. This indicates that $\mathcal{C}(S)$ assigns a more distinctive values than $\mathcal{S C}_{d}(S)$ measure, making their separation capacity bigger.

Figure 3 shows some examples of $3 D$ shapes of different kinds of objects, ordered according to their $\mathcal{C}(S)$ measure 1 . Results are in accordance with our perception. E.g. shapes (f) and (g) are ranked very closely. This is understandable since they share some of the same features: elongated body with thin wings spread out perpendicular to the main body; therefore it would be expected that they are considered to have similar shapes; for the purpose of an image classification task, these two shapes would be very likely grouped together, which could be expected.

Figures (a) (b) and (c) are all human shapes in different positions and their measured cubeness of (b) and (c) are close to each other, however and gets a higher score because of the position of legs and arms.

Shape (h) gets a very low $\mathcal{C}(S)$ measure, due to the fact that, having a long tail and neck, the dinosaur haves a very elongated shape, which greatly differs from a cube. Compare it with the horse in (d) the horse has a much shorter neck and tail, therefore we would expect the horse to have a higher cubeness than the dinosaur, as it is the case. However the horse still has a long neck, and long and thin legs, which make his cubeness measure relatively low.

The cubeness measure $\mathcal{C}(S)$ provides some classification power which may be combined with other shape descriptors in order to correctly classify shapes. However cubeness values are not unique for any shape (with the exception of a perfect cube which will always have $\mathcal{C}(S)=1$ ); similar shapes will produce similar values, but other non-similar shapes could also produce similar values. Thus it is possible, for example, to have shapes with $\mathcal{C}(S)$ scores similar to (b) but which does not look like (b). This is unavoidable and is also the reason why different shape descriptors must be combined with to achieve better classification power.

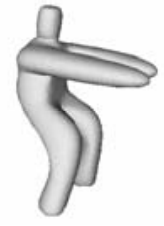

(a) 0.693

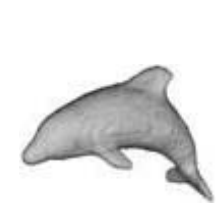

(e) 0.167

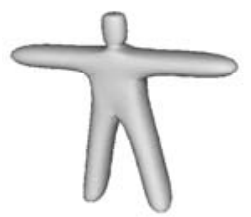

(b) 0.631

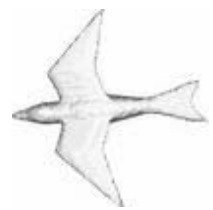

(f) 0.137

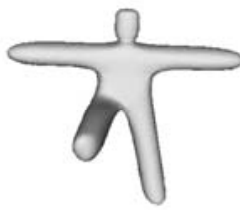

(c) 0.614

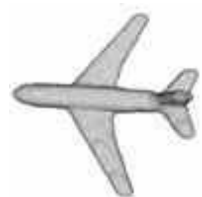

(g) 0.132

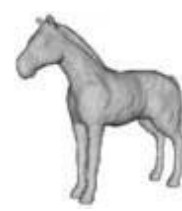

(d) 0.176

Fig. 3. Different shapes ordered according to their cubeness $\mathcal{C}(S)$

\footnotetext{
${ }^{1}$ Some of the shapes are taken from the McGill database: http://www.cim.mcgill.ca/ shape/benchMark/
} 
Figure 4 shows a cube with different levels of erosion. Values of $\mathcal{C}(S)$ decrease as more and more pieces of the shape are removed. As the level of erosion increases and the shape looks less like a cube, the produced $\mathcal{C}(S)$ values drop. Small levels of erosion do not affect too much the produced values. Such a behaviour with respect to the erosion could also suggest the robustness of $\mathcal{C}(S)$ with respect to noise. Such a robustness is expected because $\mathcal{C}(S)$ is "volume based" measure - i.e. takes into account all points inside a shape.

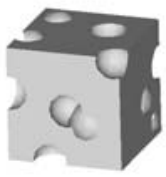

(a) 0.969

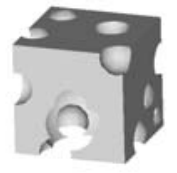

(b) 0.853

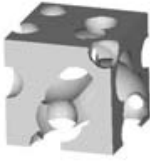

(c) 0.717

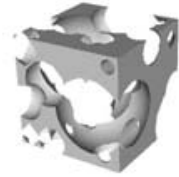

(d) 0.557

Fig. 4. Measured cubeness $\mathcal{C}(S)$ of a cube with different levels of erosion

Figure 5 illustrates some advantages that the new measure $\mathcal{C}(S)$ has over $\mathcal{C}_{d}(S)$. Each image displayed is composed of the same number of voxels $(512)$ but the voxels are in different mutual positions in each image. As it can be seen $\mathcal{C}_{d}(S)$ decreases more rapidly, as the total surface area of all appearing components increase. This is in accordance with (1). It is easy to conclude (see (11)) that $\mathcal{C}_{d}(S)$ does not depend on the mutual position of such components, but just of their surfaces. On the other side, the new measure $\mathcal{C}(S)$ takes into account the mutual positions (see Definition 1 and 10). This is an advantage. Indeed, $\mathcal{C}_{d}(S)=0$ holds for all shapes $S$ where all voxels are separated, regardless of the mutual distances between them. On the other hand, $\mathcal{C}(S)$ would produce different non-zero values which vary depending on the mutual voxel positions.

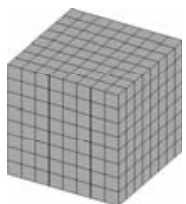

(a) 1.000

(1.000)

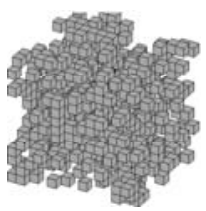

(f) 0.552

(0.199)

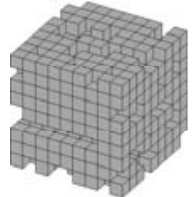

(b) 0.884

(0.715)

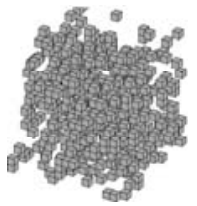

(g) 0.473

$(0.113)$

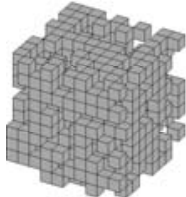

(c) 0.800 (0.526)

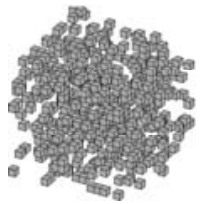

(h) 0.436

(0.084)

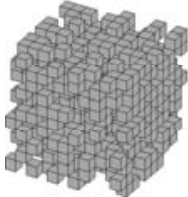

(d) 0.722

(0.387)

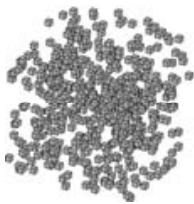

(i) 0.319

(0.036)

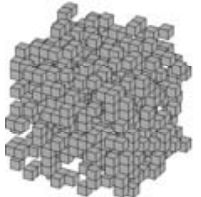

(e) 0.606

(0.234)

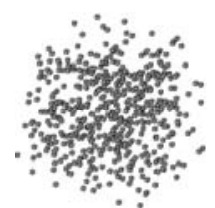

(j) 0.205

(0.007)

Fig. 5. Same number of voxels spread over different volumes. The measured $\mathcal{C}(S)$ are immediately below the shapes while $\mathcal{C}_{b}(S)$ values are in brackets. 


\section{Conclusion}

In this paper we introduce a $3 D$ shape measure, cubeness, $\mathcal{C}(S)$ defined as:

$$
\mathcal{C}(S)=\frac{3}{8} \cdot \frac{\operatorname{Volume}(S)^{4 / 3}}{\min _{\alpha, \beta \in[0,2 \pi]} \iiint_{S(\alpha, \beta)} \max \{|x|,|y|,|z|\} d x d y d z}
$$

The new measure has several desirable properties: it ranges over $(0,1]$; it gives measured cubeness of 1 if and only if the given shape is a cube; and it is invariant with respect to translation, rotation and scaling. Several experiments are given to illustrate the behaviour of the new measure. The experimental results are in accordance with theoretical considerations and with our perception. The measure works in both the discrete and continuous space, contrary to $\mathcal{C}_{d}(S)$ (from [2]) which is only applicable to voxelized data.

\section{References}

1. Bribiesca, E.: A measure of compactness for 3d shapes. Comput. Math. Appl. 40, 1275-1284 (2000)

2. Bribiesca, E.: An easy measure of compactness for $2 \mathrm{~d}$ and $3 \mathrm{~d}$ shapes. Pattern Recognition 41, 543-554 (2008)

3. Funkhouser, T., Min, P., Kazhdan, M., Chen, J., Halderman, A., Dobkin, D., Jacobs, D.: A Search Engine for 3D Models. ACM Transactions on Graphics 22(1) (2003)

4. Mamistvalov, A.G.: n-Dimensional moment invariants and conceptual mathematical theory of recognition n-dimensional solids. IEEE Trans. Patt. Anal. Mach. Intell. 20, 819-831 (1998)

5. Rahtu, E., Salo, M., Heikkila, J.: A new convexity measure based on a probabilistic interpretation of images. IEEE Trans. on Patt. Anal. and Mach. Intell. 28, 15011512 (2006)

6. Rosin, P.L.: Measuring shape: ellipticity, rectangularity, and triangularity. Machine Vision and Applications 14, 172-184 (2003)

7. Rosin, P.L.: Measuring sigmoidality. Patt. Rec. 37, 1735-1744 (2004)

8. Siddiqi, K., Zhang, J., Macrini, D., Shokoufandeh, A., Bouix, S., Dickinson, S.: Retrieving articulated $3 \mathrm{~d}$ models using medial surfaces. Machine Vision and Applications 19(4), 261-275 (2008)

9. Sonka, M., Hlavac, V., Boyle, R.: Image Processing: Analysis and Machine Vision. In: Thomson-Engineering (September 1998)

10. Stojmenović, M., Žunić, J.: Measuring elongation from shape boundary. Journal Mathematical Imaging and Vision 30, 73-85 (2008)

11. Vranić, D.V., Saupe, D.: 3d shape descriptor based on 3d Fourier transform. In: Proceedings of the EURASIP Conference on Digital Signal Processing for Multimedia Communications and Services (ECMCS 2001), pp. 271-274 (2001)

12. Žunić, J., Hirota, K.: Measuring shape circularity. In: Ruiz-Shulcloper, J., Kropatsch, W.G. (eds.) CIARP 2008. LNCS, vol. 5197, pp. 94-101. Springer, Heidelberg (2008)

13. Žunić, J., Rosin, P.L.: Rectilinearity measurements for polygons. IEEE Trans. on Patt. Anal. and Mach. Intell. 25, 1193-1200 (2003)

14. Žunić, J., Hirota, K., Martinez-Ortiz, C.: Compactness Measure for 3D Shapes (Submitted) 